\title{
Paciente con síndrome de Klippel-Trenaunay. Reporte de un caso de reemplazo articular de cadera suspendido durante la cirugía
}

\author{
Fernando A. Lopreite, Joaquín Rodríguez, Fernando Tillet, Stefano Gaggiotti, Leonardo M. Cullari, Hernán del Sel
}

Servicio de Ortopedia y Traumatología, Hospital Británico de Buenos Aires, Ciudad Autónoma de Buenos Aires, Argentina

\section{RESUMEN}

El síndrome de Klippel-Trenaunay es un trastorno congénito infrecuente. Por lo general, afecta uno de los miembros inferiores. Los hallazgos típicos son angiomas, malformaciones venosas, várices hipertróficas con comunicaciones arteriovenosas e hipertrofia del miembro afectado que, en algunos casos, altera la estructura ósea. El objetivo de esta presentación es describir el caso de un paciente de 54 años en el que se programó un reemplazo articular de la cadera izquierda durante un año y la cirugía debió suspenderse por el riesgo de exanguinación, y analizar la bibliografía al respecto.

Palabras clave: Síndrome de Klippel-Trenaunay; malformaciones arteriovenosas, sangrado masivo, cadera.

Nivel de Evidencia: IV

Patient with Klippel-Trenaunay Syndrome. Case Report of an Intraoperatively Aborted Hip Joint Replacement

\section{ABSTRACT}

Klippel-Trenaunay Syndrome is a rare congenital disorder. It usually affects one of the lower limbs. It is characterized by the presence of angiomas, venous malformations, hypertrophic varices with arteriovenous communications and hypertrophy of the affected limb, altering in some cases the bone structure. The aim of this presentation is to describe the case of a 54-year-old patient for whom a joint replacement of the left hip had been scheduled for one year and the surgery had to be aborted due to the risk of exsanguination of the patient, and to analyze the literature in this regard.

Key words: Klippel-Trenaunay syndrome; arteriovenous malformations; massive bleeding; hip.

Level of Evidence: IV

\section{INTRODUCCIÓN}

El síndrome de Klippel-Trenaunay es un trastorno congénito infrecuente, descrito en 1900, por médicos franceses. ${ }^{1}$ Por lo general, afecta uno de los miembros inferiores. Los hallazgos característicos son angiomas, malformaciones venosas, várices hipertróficas con comunicaciones arteriovenosas e hipertrofia del miembro afectado.

En algunos casos, la malformación compromete la estructura ósea y genera comunicaciones arteriovenosas intraóseas con alteración de la arquitectura del hueso. Se menciona a la hemartrosis recurrente como la causa de la degeneración articular.

Hasta ahora se han publicado cuatro pacientes con compromiso de la cadera a quienes se les programó un reemplazo articular..$^{2-5}$ Uno de ellos debió suspenderse a causa del riesgo de exanguinación. ${ }^{5}$

El objetivo de este reporte es presentar un caso similar al descrito, en un paciente de 54 años a quien se le programó un reemplazo articular de cadera izquierda durante un año. La cirugía debió suspenderse por el riesgo intraoperatorio de exanguinación del paciente.

Recibido el 2-5-2020. Aceptado luego de la evaluación el 30-5-2020 • Dr. FERNANDO A. LOPREITE • fernandolopreite@ hotmail.com

https://orcid.org/0000-0002-2065-8649

Cómo citar este artículo: Lopreite FA, Rodríguez J, Tillet F, Gaggiotti S, Cullari LM, del Sel H. Paciente con síndrome de Klippel-Trenaunay. Reporte de un caso de reemplazo articular de cadera suspendido durante la cirugía. Rev Asoc Argent Ortop Traumatol 2021;86(2):240-245. https://doi.org/10.15417/issn. 1852-7434.2021.86.2.1108 


\section{CASO CLÍNICO}

Paciente de 54 años con diagnóstico de síndrome de Klippel-Trenaunay que afecta el miembro inferior izquierdo y artrosis de la cadera homolateral de cinco años de evolución (Figura 1). El miembro inferior izquierdo tiene un diámetro mayor que el contralateral, y se detectan múltiples cordones varicosos con latido palpable en la ingle, el tobillo y la región glútea posterior (Figura 2). El paciente camina con bastón y la cadera en flexión y hay acortamiento del miembro. Presenta escoliosis lumbar y lumbalgia; la rodilla homolateral no está afectada.

A
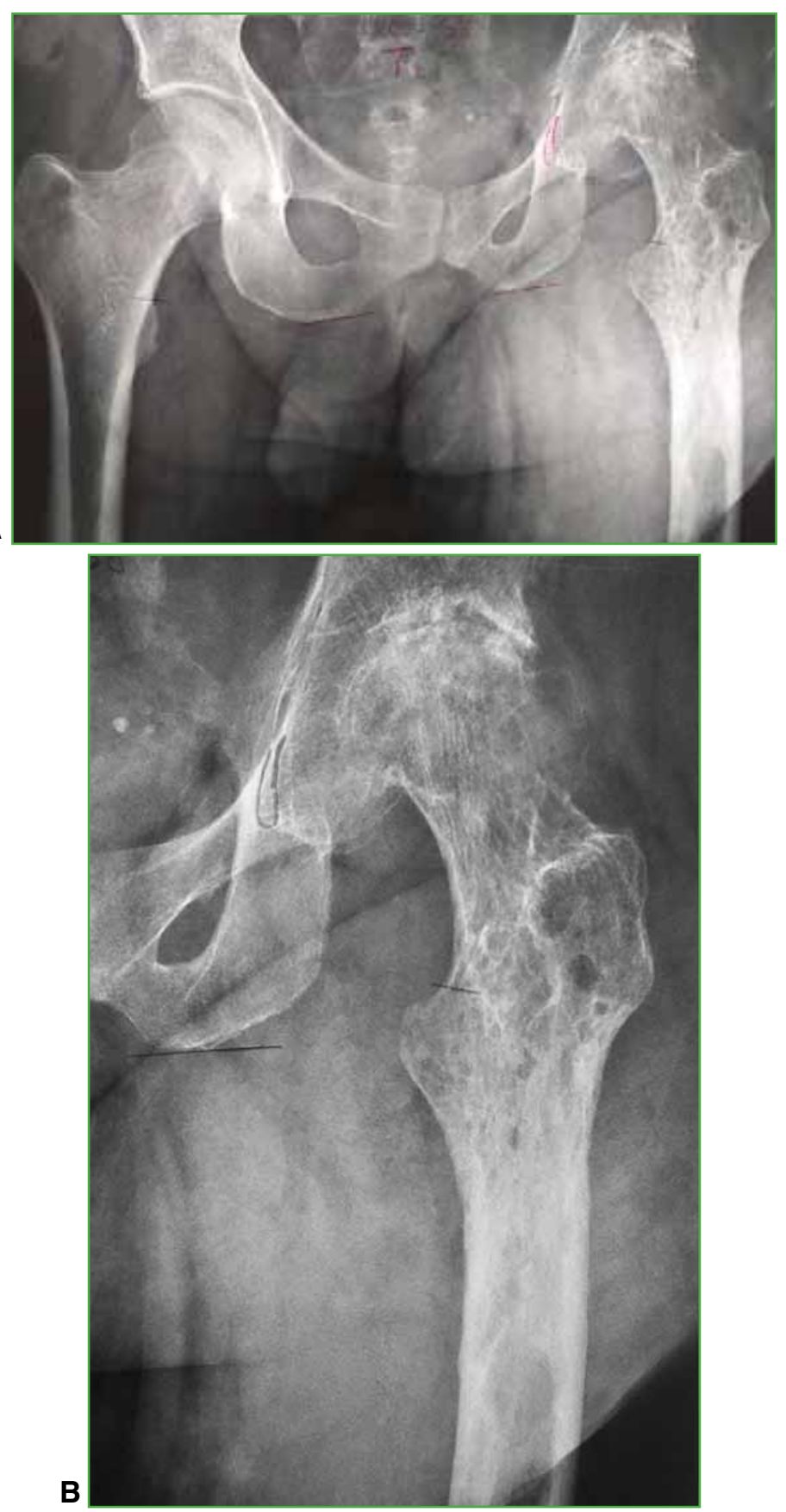

Figura 1. A. Radiografía de ambas caderas, de frente. B. Radiografía de cadera izquierda, de frente. Se observa la cadera izquierda artrósica, con imágenes líticas en la cabeza, el cuello y la región pertrocantérica que corresponderían a las anastomosis arteriovenosas. 

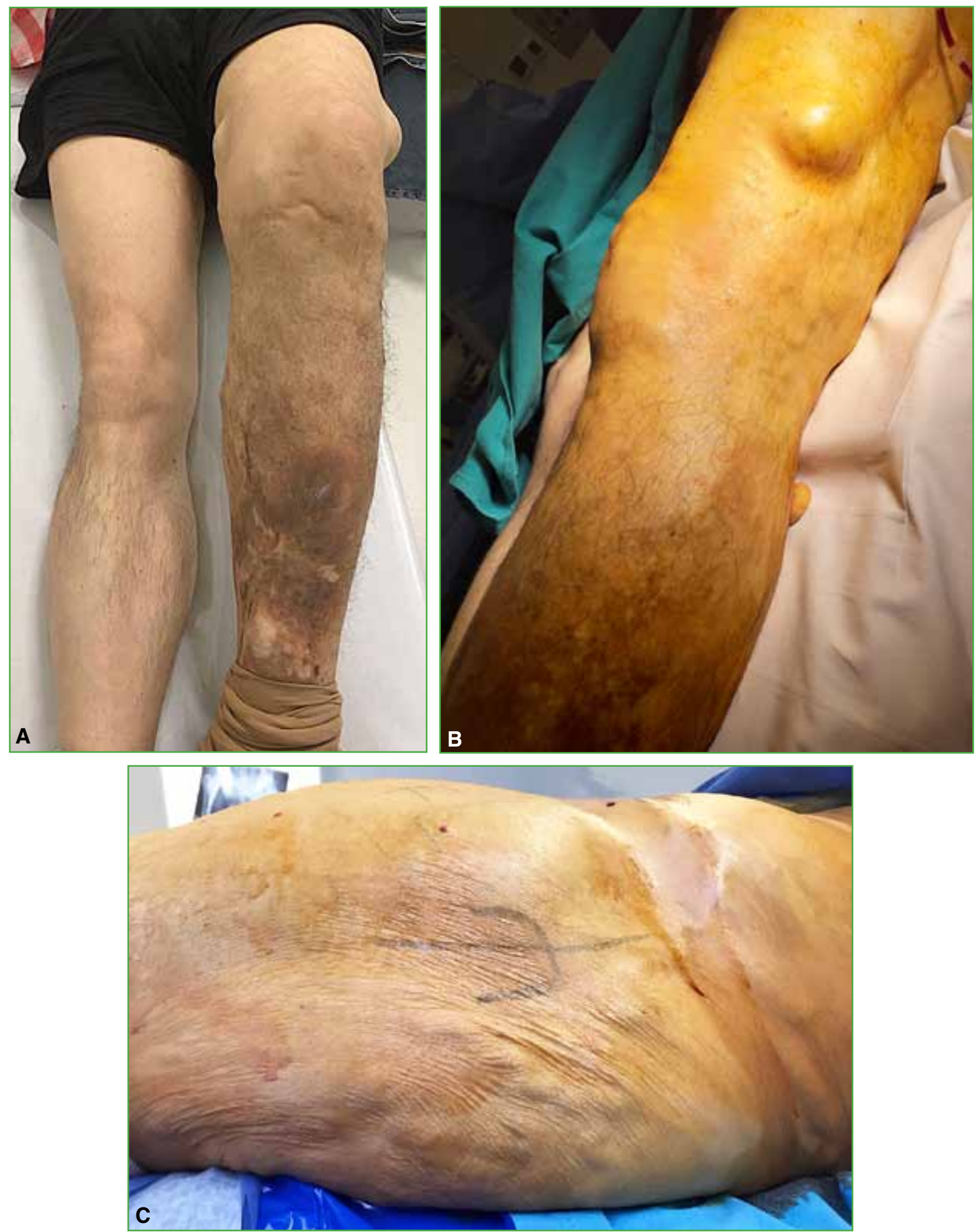

Figura 2. A y B. Imagen del miembro inferior izquierdo. Se puede observar el cambio en la coloración de la piel de la pierna y tumoraciones en las caras externa e interna del muslo que corresponden a las anastomosis arteriovenosas. C. Se observa la región de la cadera por operar. Están marcados el trocánter mayor y la incisión por vía anterolateral con el paciente en decúbito dorsal. Se observa un terreno varicoso en la región glútea que dificultaba el abordaje posterolateral. 
El paciente fue sometido a arteriografías y ecodoppler a cargo de equipo de Cirugía Cardiovascular del hospital. Se definió que la región lateral era la mejor para abordar la cadera (Figura 2), pues no había formaciones varicosas ni anastomosis arteriovenosas detectables clínicamente ni en los estudios.

Se realizó una resonancia magnética en la que se constató la alteración de la arquitectura ósea por malformaciones vasculares en la cabeza, el cuello y la metáfisis femoral (Figura 3).

Se programó la cirugía mediante un abordaje anterolateral directo, con suplementos sanguíneos y unidades de sangre disponibles para el procedimiento. El paciente estaba anticoagulado, por lo que el Equipo de Hemoterapia suspendió dicha profilaxis antes de la cirugía.

Se programó la colocación de una prótesis total de cadera híbrida, cotilo no cementado y tallo cementado, considerando la arquitectura del fémur proximal (Figura 1) y que, en la resonancia magnética (Figura 3), se observaban múltiples imágenes intraóseas correspondientes a fístulas arteriovenosas. La idea era disminuir el sangrado intracanal con el cementado.
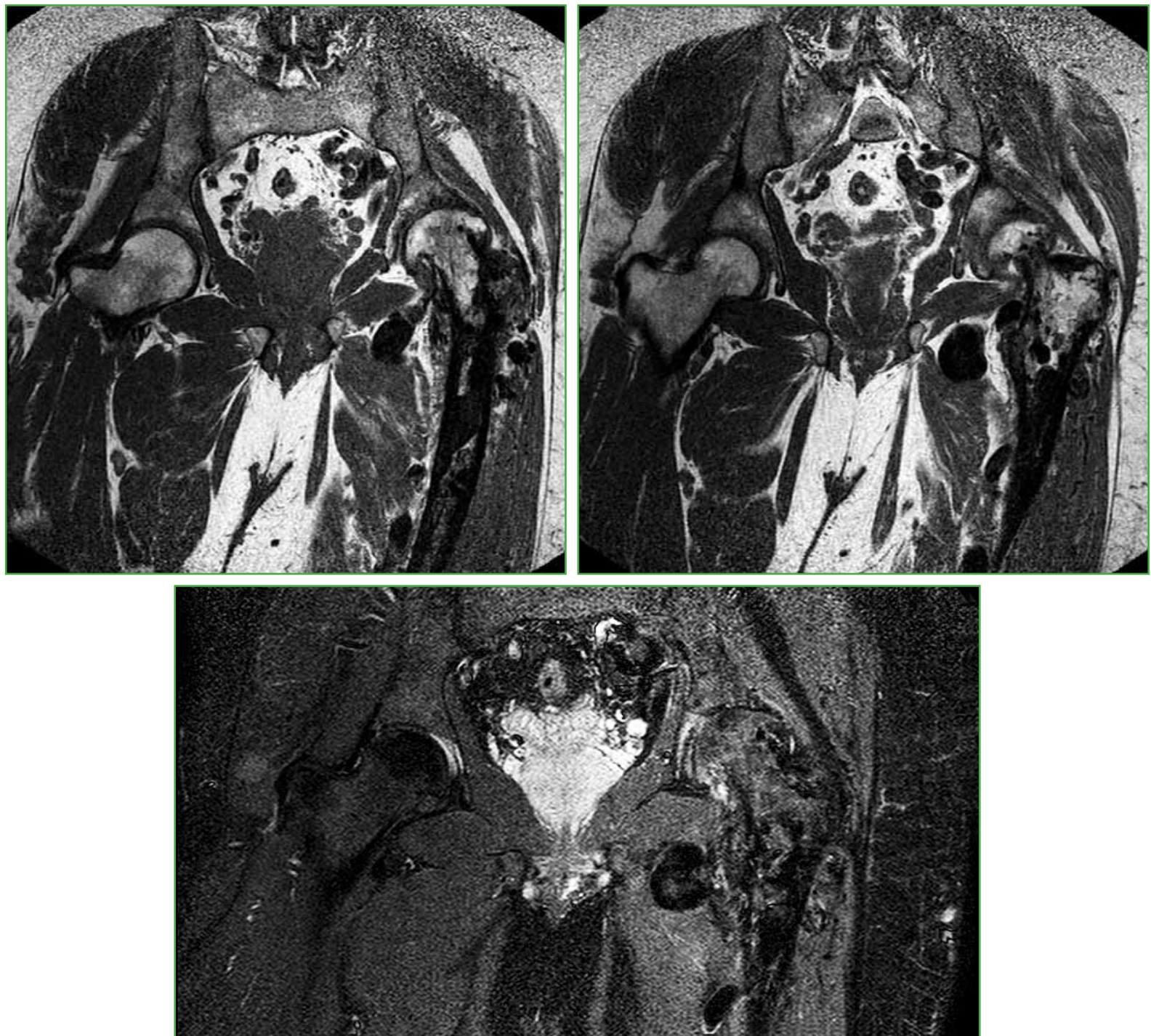

Figura 3. Resonancia magnética, cortes coronales. Se pueden verificar los islotes sanguíneos, sobre todo, en la región pertrocantérica con alteración en la arquitectura ósea. 
$\mathrm{Al}$ realizar el abordaje, en el tejido celular subcutáneo, se produjo un abundante sangrado por dos formaciones venosas de $1 \mathrm{~cm}$ de diámetro cada una que debieron ser ligadas. Durante la capsulotomía, desde los agujeros nutricios del cuello femoral, se produjo un intenso sangrado difícil de cohibir. En ese momento, ya se habían acumulado 1,5 litros de sangre en el frasco de aspiración. Se consulta con los cirujanos vasculares que estaban en el quirófano contiguo, ya que se preveía que, al realizar la osteotomía del cuello femoral, el sangrado podría ser cataclísmico e incoercible por las malformaciones vasculares intraóseas, y se decide, en conjunto, suspender el procedimiento.

El paciente debió ser transfundido durante la cirugía y en el posoperatorio inmediato. Se recuperó favorablemente y fue dado de alta a las 72 horas.

\section{DISCUSIÓN}

Según los cuatro artículos publicados, en tres casos, fue posible llevar a cabo la artroplastia total de cadera programada y todos tuvieron un importante sangrado..$^{2-4}$

En el cuarto paciente, el procedimiento fue suspendido en el mismo momento que en el nuestro, al hacer la capsulotomía, por el riesgo de exanguinación, y se identificaron múltiples fuentes de sangrado intraóseas que no se habían detectado en la arteriografía. ${ }^{5}$ Cabe aclarar que ese intento fue el tercero; según los autores, se había modificado la vía de abordaje por una anterior, porque había menos fístulas arteriovenosas.

Analizando las radiografías de cadera de los casos publicados, en los tres pacientes en quienes se completó el reemplazo articular, la alteración intraósea no era tan significativa, como en la que debió suspenderse el procedimiento y en el nuestro (Figura 1).

Efectuar un abordaje cuidadoso con disección de los vasos con ligaduras puede limitar el sangrado, pero es posible que no se puedan manejar las malformaciones intraóseas durante la cirugía, por lo que el riesgo de sangrado incoercible es alto. Por este motivo, es importante contar con suplementos sanguíneos y sangre para reponer, y en lo posible, recuperador celular.

En estos pacientes, los episodios tromboembólicos son frecuentes, con riesgo de embolia pulmonar (14\%) y trombosis venosa profunda (16\%) ${ }^{6,7}$ Nuestro paciente estaba anticoagulado; por lo tanto, fue preciso regularizar los valores de coagulación antes de la cirugía.

Otro tema por considerar es el implante que se colocará, ya que la alteración de la arquitectura ósea puede ser considerable. En el caso publicado por Mallick y cols., ${ }^{2}$ se realizó una artroplastia total cementada en un paciente de 58 años para disminuir el sangrado óseo. García-Juárez y cols. ${ }^{4}$ utilizaron un implante no cementado, y señalan que no haber utilizado un implante cementado pudo ocasionar el sangrado posoperatorio del paciente, que tuvo un nivel de hemoglobina de $4 \mathrm{mg}$ al tercer día de la cirugía, y recién fue posible lograr la estabilidad hemodinámica con transfusiones el día 14 del posoperatorio, con una hemoglobina de $9 \mathrm{mg}$, y recibió el alta a los 23 días. En nuestro caso, la idea era colocar una prótesis híbrida, con un tallo cementado pensando en la mala calidad ósea femoral y en las fístulas arteriovenosas observadas en las radiografías y en la resonancia magnética de fémur proximal y, de esta manera, obturar el hueso sangrante con el cemento. Con seguridad, el cementado sería de mala calidad y podría alterar la supervivencia del implante.

\section{CONCLUSIONES}

Estos pacientes tienen un alto riesgo de sangrado y, en consecuencia, deben ser cuidadosamente estudiados. La vía de abordaje se debe planificar adecuadamente y se debe estar preparado para la ligadura de grandes vasos venosos.

Asimismo, es fundamental identificar la magnitud de las malformaciones intraóseas, ya que, de ello, podrá depender si se lleva a cabo la cirugía o no y el tipo de fijación del implante, teniendo en cuenta que, una vez realizada la osteotomía del cuello femoral, el sangrado puede ser incontrolable y poner en riesgo la vida del paciente.

Coincidimos con Cirstoiu y cols. ${ }^{5}$ en la recomendación del manejo no quirúrgico de estos pacientes, mientras sea posible.

Conflicto de intereses: Los autores no declaran conflictos de intereses.

ORCID de J. Rodríguez: https://orcid. org/0000-0002-1089-3071 ORCID de F. Tillet: https://orcid.org/0000-0003-2658-9856 ORCID de S. Gaggiotti: https://orcid.org/0000-0003-4077-2892
ORCID de L. M. Cullari: https://orcid.org/0000-0002-6058-6686 ORCID de H. del Sel: https://orcid.org/0000-0002-3655-1408 


\section{BIBLIOGRAFÍA}

1. Klippel M, Trenaunay P. Du naevus variqueux ostéohypertrophique. Arch Gen Med (París) 1900;185(3):641-72.

2. Mallick A, Weber A. An experience of arthroplasty in Klippel-Trenaunay syndrome. Eur J Orthop Surg Traumatol 2007;17:97-9. https://doi.org/10.1007/s00590-006-0114-z

3. Willis-Owen CA, Cobb JP. Total hip arthroplasty in Klippel-Trenaunay syndrome. Ann R Coll Surg Engl 2008;90(8):W6-8. https://doi.org/10.1308/147870808X303182

4. García-Juárez JD, Tohen-Bienvenu A, Jimenez-Cabuto IC, Molina-Méndez J. Artroplastía total de cadera en el síndrome de Klippel-Trenaunay. Reporte de un caso y revisión de la literatura. Acta Ortop Mexicana 2011;25(2):126-9. Disponible en: https://www.medigraphic.com/pdfs/ortope/or-2011/or112j.pdf

5. Cirstoiu C, Cretu B, Sandu C, Dorobat B, Neagu A, Serban B. Failed attempt of total hip arthroplasty in a patient with Klippel-Trenaunay syndrome. JBJS Case Connect 2019;19(4):1-5. https://doi.org/10.2106/JBJS.CC.19.00103

6. Baskerville PA. Tromboembolic disease and congenital venous abnormalities. Phlebologie 1987;40:531-6. PMID: 3039545

7. Muluk SC, Ginns LC, Semigram MJ, Kaufman JA, Gertler JP. Klippel-Trénaunay syndrome with multiple pulmonary emboli-an unusual cause of progressive pulmonary dysfunction. J Vasc Surg 1995;21:686-90. https://doi.org/10.1016/s0741-5214(95)70199-0 